\title{
En-face optical coherence tomography analysis of gold and silver nanoparticles in endodontic irrigating solutions: An in vitro study
}

\author{
FLORIN TOPALA $^{1 *}$, LUMINITA-MARIA NICA $^{2}$, MARIUS BOARIU $^{2 *}$, MEDA LAVINIA NEGRUTIU $^{3}$, \\ COSMIN SINESCU ${ }^{3}$, ADRIAN MARINESCU ${ }^{2}$, LAURA ELENA CIRLIGERIU ${ }^{2}$, STEFAN-IOAN STRATUL $^{4}$, \\ DARIAN RUSU ${ }^{4 *}$, RENATO CHINCIA $^{5}$, VIRGIL FLORIN DUMA ${ }^{6}$ and ADRIAN PODOLEANU ${ }^{7}$ \\ 13rd Department, Discipline of Prosthetic Dentistry, Faculty of Dental Medicine, 'Victor Babes' University of Medicine \\ and Pharmacy; ${ }^{2} 3$ rd Department, Discipline of Restorative Dentistry and Endodontics, Research Center TADERP, \\ Faculty of Dental Medicine, 'Victor Babes' University of Medicine and Pharmacy; ${ }^{3} 1$ st Department, Discipline of \\ Prosthesis Technology and Dental Materials, Faculty of Dental Medicine, 'Victor Babes' University of Medicine and \\ Pharmacy; ${ }^{4}$ st Department, Discipline of Periodontology, Faculty of Dental Medicine, 'Victor Babes' University of \\ Medicine and Pharmacy, 300070 Timisoara; ${ }^{5}$ Private Practice, 110357 Pitesti; ${ }^{6}$ Department of Product Design and AAMR, \\ Faculty of Engineering, 'Aurel Vlaicu' University, 310130 Arad, Romania; ${ }^{7}$ Applied Optics Group, \\ School of Physical Sciences, University of Kent, Canterbury CT2 7NH, UK
}

Received April 9, 2021; Accepted May 10, 2021

DOI: $10.3892 / \mathrm{etm} .2021 .10424$

\begin{abstract}
Optical coherence tomography (OCT) is a non-invasive, non-radioactive optical diagnostic method based on low-coherence interferometry, which achieves images with different orientation. In dentistry, its major advantage is represented by the localization and characterization of the smallest defects in hard dental tissues, dental materials and of the smallest details in dental anatomy (supplementary canals, recesses, isthmuses, or intra-radicular connections). The aim of the present in vitro study was to evaluate using c-scan en-face optical coherence tomography, the optical opacity, and the distribution inside the root canal lumen of several extracted human teeth of silver and gold nanoparticles from special irrigating solutions used in endodontic treatment. Twelve root canals from 5 human teeth were instrumented using the ProTaper Universal system after initial negotiation with hand K-files ISO no. 10 and rotary nickel-titanium PathFile instruments. An initial c-scan OCT analysis was performed for each sample to confirm that the root canal lumen was empty from radiopaque materials (Group 1). Teeth were first irrigated with NanoCare Plus (Group 2) and then with NanoCare Gold (Group 3) and C-scans were repeated after each irrigation method. The OCT investigation started at
\end{abstract}

Correspondence to: Dr Luminita-Maria Nica, 3rd Department, Discipline of Restorative Dentistry and Endodontics, Research Center TADERP, Faculty of Dental Medicine, 'Victor Babes' University of Medicine and Pharmacy, 9 Revolutiei 1989 Blv., 300070 Timisoara, Romania

E-mail: luminita.nica98@yahoo.ro

*Contributed equally

Key words: optical coherence tomography, gold nanoparticles, silver nanoparticles, endodontics, nanotechnology the tooth apex, at a depth of $1 \mathrm{~mm}$ from its tip. Subsequently, 100 slices of 10 microns were obtained from each root canal. Images were captured and then analyzed with ImageJ software to calculate the level of grey inside the root canal lumen. The highest values of grey were obtained in the samples irrigated with NanoCare Gold after NanoCare Plus (Group 3). The present study proved that both nanoparticles inserted in root canal irrigants were evidenced through OCT imagistic analysis due to their optical opacity, which allowed their highlighting in an empty root canal lumen, after the endodontic treatment was performed and the root canal was cleaned and shaped using specific protocols.

\section{Introduction}

Trends pertaining to antimicrobial endodontic irrigation solutions and of nanoparticles used in root canal filling materials are focused on disinfection of the endodontic system and prevention of the development of bacterial biofilm. Since failures of endodontic treatment are present in clinical practice, there is continuous development of endodontic irrigating solutions and of root canal filling materials. More specifically, nanotechnology helps to create new materials with biologic applications, including dental biomaterials (1).

Dental nano-biomaterials are based on polymeric or metallic nanoparticles, with demonstrated antimicrobial effect, which are incorporated in filling materials or are used as adjuvants in root canal cleaning to eliminate the presence of microorganisms (2). Silver colloid is of special interest because of its distinctive properties as good conductivity, chemical stability, and catalytic and antibacterial activity. Silver diamine fluoride $\left[\mathrm{Ag}\left(\mathrm{NH}_{3}\right)_{2} \mathrm{~F}\right]$ solution has been used in the prevention and progression of dental caries. When silver diamine fluoride is used at a concentration of $3.8 \%$ as a root canal irrigant or as interim medication, it has potential activity in the reduction of bacterial adhesion (3). 
Silver colloid is considered a 'natural antibiotic' from antiquity, being non-toxic for human cells, but highly toxic for some bacterial species, such as E. coli, Staphylococcus aureus, and others (2). It is an aqueous solution of ultra-small silver nanoparticles, with 1-9 nanometers diameter in distilled water, with the antimicrobial activity of silver colloid being very similar to that of antibiotics with large spectrum. Its presence activates an enzyme with local action against each bacteria, virus, and fungi. Silver colloid establishes the acid-base equilibrium and initiates external resistance. At this colloid, polymorph or mutant microorganism is extremely sensitive (2).

Gold nanoparticles are generally 1-100 nm particles usually suspended in a fluid (colloidal gold). Known as Nanogold, colloidal gold suspension is usually burgundy red (for spherical gold nanoparticles with a diameter $<100 \mathrm{~nm}$ ), or yellow brown (for higher diameter of the nanoparticles). The diameter of nanoparticles plays an indispensable role in nanotechnology and is crucial for all biological applications. In recent years, gold nanoparticles have been intensely studied because of their optical properties, with large applications in biomedicine (4-10). As elementary silver liberates ions and gold is known for its optical properties and the absorption of proteins, the combination of these two elements, especially in one single material, has been tested to obtain a synergistic effect of their properties. For example, the utilization of silver nanoparticles in combination with gold can create new possibilities for materials with antibacterial activity (1).

Two new irrigating solutions based on gold and silver nanoparticles have been introduced in endodontic practice: NanoCare Plus Silver Gold and NanoCare Gold (Nanotechnology Dental, Poland). NanoCare Plus Silver Gold is a pharmaceutical complex with long-lasting bacteriostatic activity, indicated as a final irrigant in root canal treatment. The aim of this solution is to complete the cleaning of the endodontic system obtained after conventional irrigation protocol. Sodium hypochlorite is used as the main endodontic irrigant as, owing to its germicide and proteolitic activity, it eliminates most of the organic substances from the root canals. Nanocare Plus Silver Gold acts as a supplementary cleaner of organic rests and because of the gold and silver nanoparticles contained, prevents the bacterial re-colonization inside the root canal system. The final step of the root canal filling is not negatively influenced by the corrosion of gold and silver nanoparticles and by their minimal concentration in the irrigating solution $(11,12)$.

NanoCare Gold was developed with the purpose of immediate use prior to filling, cementation of a prosthetic restoration, veneer or inlay/onlay, or covering the entire surface of the tooth with a layer of nanoparticles. Due to the chemical neutrality of silver and gold nanoparticles, the components of NanoCare Gold do not interact with the products usually used in dental treatment, thus, their properties are preserved $(11,12)$. Moreover, the nanosilver and nanogold particles from its structure do not deactivate by the light used in photo polymerization of the restoration. NanoCare Gold has a positive influence on the adhesion of composite materials to dentin, which increases the longevity of the restoration (a lower risk of micro-fissure occurrence). It is very important that NanoCare Gold does not change the color of the final restoration $(11,12)$.

Optical Coherence Tomography (OCT) was first reported as a new imagistic method by Huang et al (13) in 1991. Since then, it has a lot of clinical applications in medical diagnosis, including the field of dentistry. OCT is a non-invasive, non-radioactive optical diagnostic method based on low-coherence interferometry, which achieves images with different orientation, with a depth resolution of $10 \mu \mathrm{m}$; after their importation in numerical simulation softs, it creates a three-dimensional reconstruction and interpretation of the analyzed structures. In dentistry, the major advantage of OCT is represented by the localization and characterization of the smallest defects in hard dental tissues or dental materials, and of the smallest details in dental anatomy, such as supplementary canals, recesses, isthmuses, or intra-radicular connections. Structural defects existent in the root canal filling material, rests of materials or debris, even at a level of nanoparticles, present in the canal lumen can be observed by using this method. Another advantage of this method is that it is total non-invasive and does not deteriorate the analyzed samples (12-15).

The aim of the present in vitro study was to evaluate using c-scan en-face optical coherence tomography (OCT) the optical opacity and the distribution inside the root canal lumen of several extracted human teeth, of silver and gold nanoparticles from special irrigating solutions used in endodontic treatment.

\section{Materials and methods}

Materials. Twelve root canals with different apical curvature from 5 human teeth extracted for periodontal reasons, after informed consent of the patients was obtained (1 monoradicular-1 canal, 1 first upper premolar-2 canals, 1 upper molar-3 canals and 2 lower molars-each with 3 canals) were instrumented using the ProTaper Universal system (Dentsply Maillefer) after initial negotiation with hand K-files ISO \#10 and rotary nickel-titanium (NiTi) PathFile instruments (Dentsply Maillefer). The root canals have been shaped in a crown-down technique, using the following working sequence of the ProTaper system: S1-S2-F1-F2-F3, at $300 \mathrm{rpm}$ and torque settings according to the manufacturer's instructions, using a conventional irrigation protocol, with common use in clinical endodontic practice. The main irrigant used for each root canal during shaping procedures was sodium hypochlorite $\mathrm{NaOCl} 5.25 \%(10 \mathrm{ml})$, in alternation with ethylenediaminetetraacetic acid EDTA $17 \%$ solution $(2 \mathrm{ml})$ and distilled water. The root canals were dried with sterile paper points at the end of cleaning and shaping until they were completely dried and no irrigant remained inside. Periapical radiographs (Fig. 1) were taken for each of the root canals included in the study to confirm the quality of the endodontic preparation.

Evaluation of root canals. A Time Domain Optical Coherence Tomography (TD-OCT) setup with the new dynamic focus method was employed for this evaluation. The output optical power used by the system was between 1 and $3.5 \mathrm{~mW}$, using a Thorlab PM100 device. The system was working in the range of 1,250-1,360 nm (OSA) (model no. HP70950B) and the width of the optical band was $>25 \mathrm{~nm}$ (OSA) (model no. HP70950B). The dynamic focus method requires only that the object arm (not the reference arm) is placed on the translation stage. By allowing physical separation of the two interferometer arms, the dynamic focus method removes a design constraint which restricts the layout of more complex systems such as those 

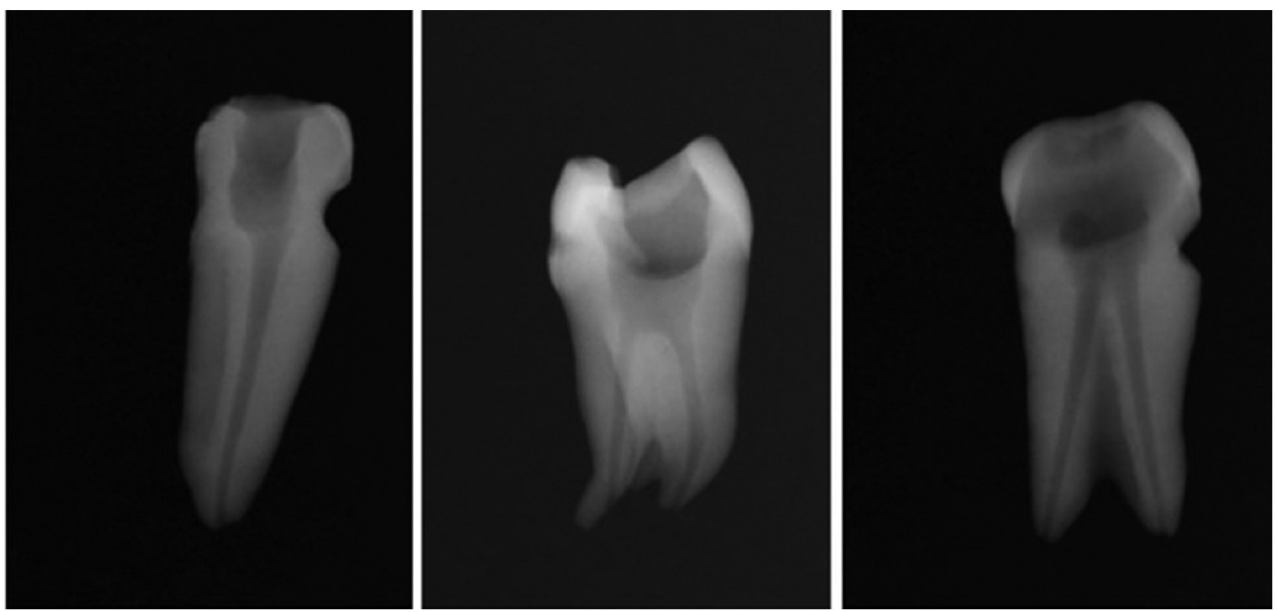

Figure 1. Initial radiographs of some of the samples after conventional endodontic treatment.
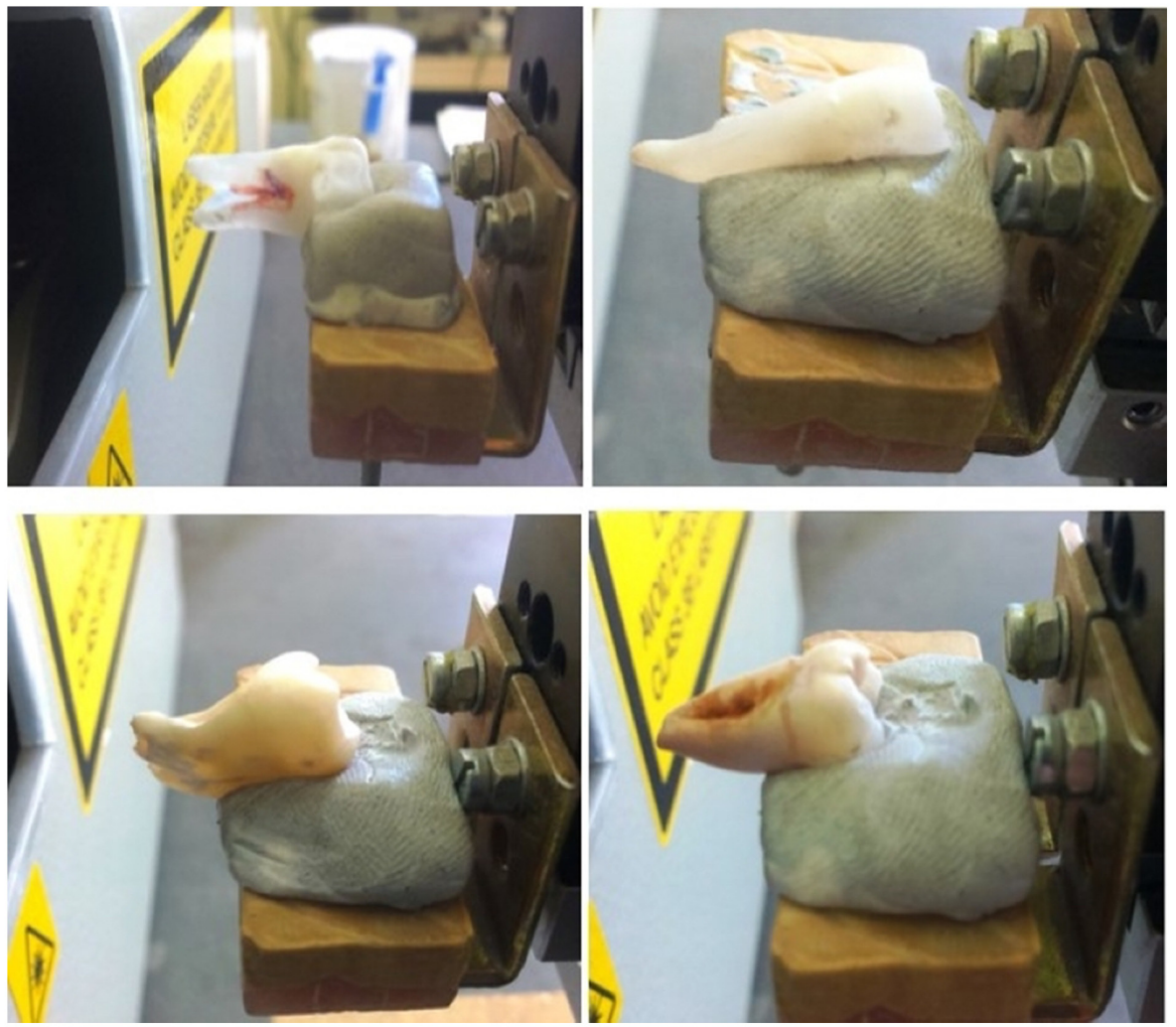

Figure 2. Some of the samples fixed for the OCT analysis.

employing adaptive optics. In addition, it permits the design of a probe-head which is both smaller and can be positioned independently of the rest of the OCT system. The TD-OCT system operates both $\mathrm{C}$-scan (en-face) and B-scan (cross-sectional) sections.

Grouping. An initial C-scan OCT analysis was performed for each of the samples to confirm that the root canal lumen is empty from radiopaque materials: Group 1 (Control group) (Fig. 2). The C-scan OCT parameters used were: $1,300 \mathrm{~nm}, 100$ sections $/ 1 \mathrm{~mm}$, with a distance of $0.01 \pm 0.005 \mathrm{~mm}$. The samples were locked in fixed positions in a block of putty silicone and placed at $20.5 \mathrm{~mm}$ from the OCT lens (Fig. 2). The C-scan OCT investigation started at the apex of the tooth, at a depth of $1 \mathrm{~mm}$ from its tip. Then, 100 slices of 10 microns were obtained.

The same 12 samples were used as two other study groups, according to the non-conventional irrigating protocol that followed, by using the nanoparticle irrigating solutions. In Group 2 ( $\mathrm{n}=12$ ), only $1 \mathrm{ml}$ of NanoCare Plus Silver Gold (Dental Nanotechnology Company) was used as supplementary irrigation for each sample (Fig. 3). The irrigant was placed into the root canal with a 25-Gauge needle introduced on the entire working length. No aspiration followed, and teeth were evaluated with another OCT investigation. In Group $3(\mathrm{n}=12), 1 \mathrm{ml}$ of NanoCare Gold (Dental Nanotechnology Company) was additionally used for irrigation in each sample (Fig. 3) and C-scan OCT analysis 
Table I. Levels of grey scores of the OCT scans before and after irrigation with nanoparticles.

\begin{tabular}{lrrrr}
\hline Group & Min & Max & Mean & SD \\
\hline 1 & 9 & 255 & 88.74076 & 4.87669 \\
2 & 12 & 255 & 102.94019 & 5.82824 \\
3 & 11 & 255 & 104.31445 & 2.12771 \\
\hline
\end{tabular}

OCT, optical coherence tomography; SD, standard deviation.

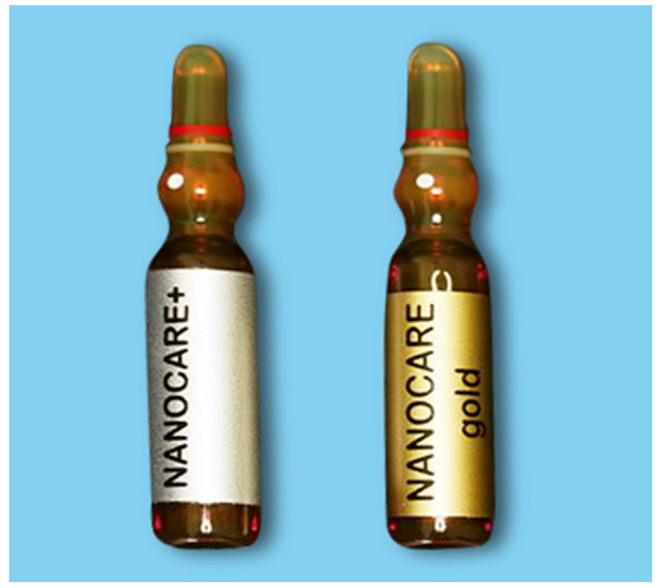

Figure 3. The nanoparticle irrigating solutions.

was again performed to determine whether there is a difference in the imagistic appearance of the levels of grey in the root canal lumen comparative to the initial OCT images (Group 1) or with the first non-conventional irrigation protocol (Group 2). The time between the irrigation and fixation and OCT evaluation of the samples was approximately $5 \mathrm{~min}$, until the OCT analysis was completed. OCT images obtained before and after the two irrigation protocols with nanoparticles solutions were compared.

Regions of interest. One hundred images representing the OCT-sections were recorded for each of the 12 root canals and were analyzed, using the public domain software ImageJ (version $1.33 \mu$; National Institute of Health), which is a free-of-charge software widely used in medical and biological image analysis over a long period of time, with a wide range of analysis functions $(16,17)$. Image files can be opened, and regions of interests (ROI) can be created automatically or by freehand selection. The ROIs can be saved and transferred to other images and edited afterwards to different projection conditions. A region of interest was opened using as its borders the margins of the root canal of every tooth. Only the root canal was enclosed in the ROI. Using the histogram tool, the pixel values of the ROIs were established. The grey values were related to the absorption of $\mathrm{X}$-rays, the radiologic density of a certain tissue. The grey values were saved in an 8-bit color space. Every pixel obtained a value between 0 and 255 in which 0 stands for black, low radiologic density and 255 for white, total X-ray absorption (Fig. 4).

Statistical analysis. The maximum, minimum, mean value and the standard deviation of the grey values were calculated.

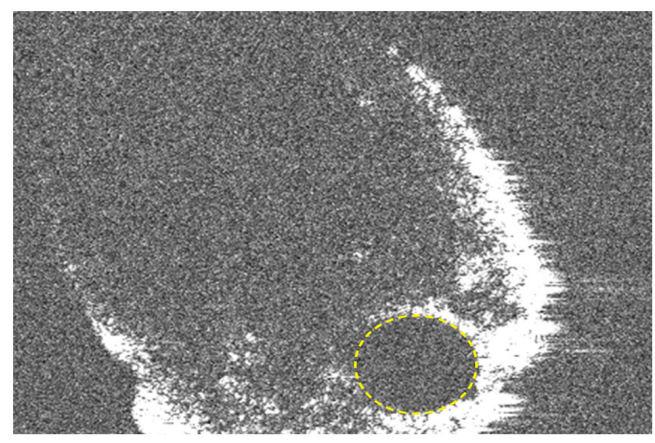

Figure 4. OCT analysis with the regions of interest (ROI) marked.

The Student's t-test was used to compare the results between the groups to identify the statistically significant differences. The result was considered statistically significant for $\mathrm{P}<0.05$.

\section{Results}

OCT analysis. The c-scan OCT analysis allowed the investigation of the root canal outline and lumen on every section that varies from 0 to $1 \mathrm{~mm}$ from the root apex. On the samples without nanoparticle-based irrigants, the root canal lumen appeared clearly separated from the dentinal contour, which is almost white on the OCT images (255 radiological density). The empty root canal lumen showed the lowest level of grey on the initial OCT images, as it was empty and almost completely black on the initial OCT-scans.

Grey values and significance. The maximum, minimum, mean value, and the standard deviation of the grey values on the analyzed OCT images for each group were recorded in Table I.

Following Student's t-test, a statistically significant difference was found between Group 1 and Group $2(\mathrm{P}<0.00001)$ and Group 1 and Group 3 ( $\mathrm{P}<0.00001)$. However, between Group 2 and Group 3 even if the data showed a difference, this was not statistically significant $(\mathrm{P}>0.05)$.

Root canal. Analysis of the images showed that the pixel values in the two groups (where nanoparticle irrigants were used) increased in comparison with the control side (where only standard sodium hypochlorite was used). The level of grey inside the root canal lumen was higher in the samples irrigated with NanoCare Plus (Group 2) compared to the initial samples (Group 1). It can be assumed that the nanoparticles present in the irrigating solution were observed as whiter, dot-like material inside the root canal. The highest levels of grey were obtained in Group 3, after the second irrigation with NanoCare Gold (Figs. 5 and 6).

The almost circular shape of the root canal contour, as the result of the rotary instrumentation with nickel-titanium instruments, was also observed (Fig. 5). On some of the analyzed samples, the eccentric position of the apical foramen in relation to the radiological apex was identified (Figs. 6 and 7).

\section{Discussion}

The OCT-based investigation of the current study revealed that nanoparticles from the irrigating solutions were observed even at the level of the last apical millimeter of the root canal, 

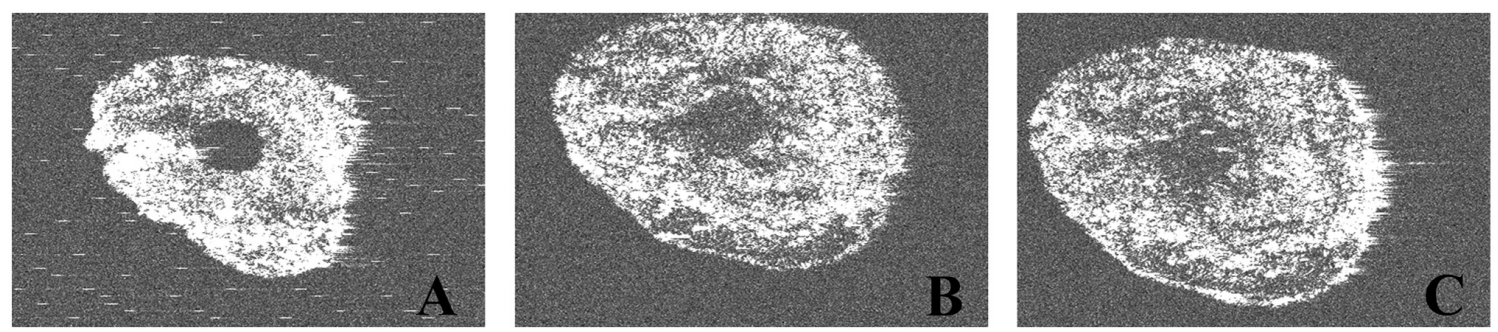

Figure 5. OCT analysis of the same section from Sample 1 before and after the non-conventional irrigation protocols. (A) Initial OCT, (B) + NanoCare Plus, (C) +NanoCare Gold.
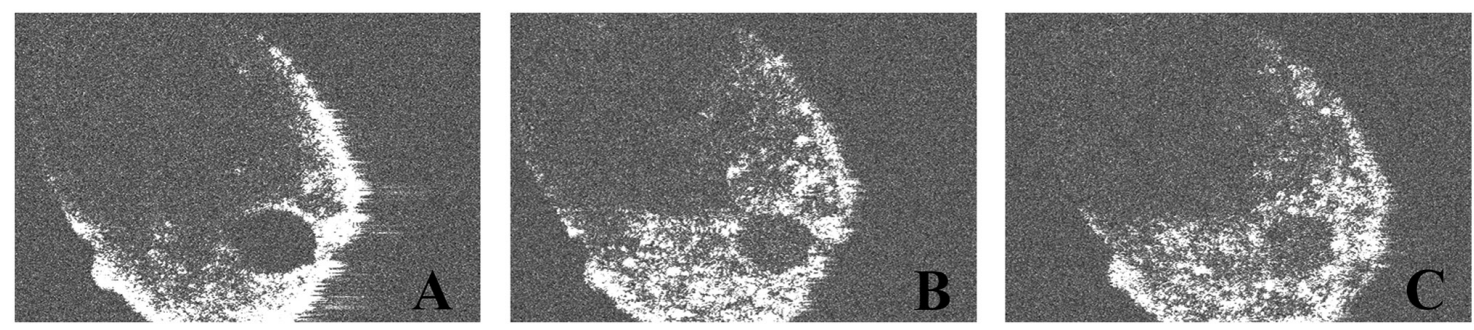

Figure 6. OCT analysis of the same section from Sample 5 before and after the non-conventional irrigation protocols. (A) Initial OCT, (B) + NanoCare Plus, (C) +NanoCare Gold.
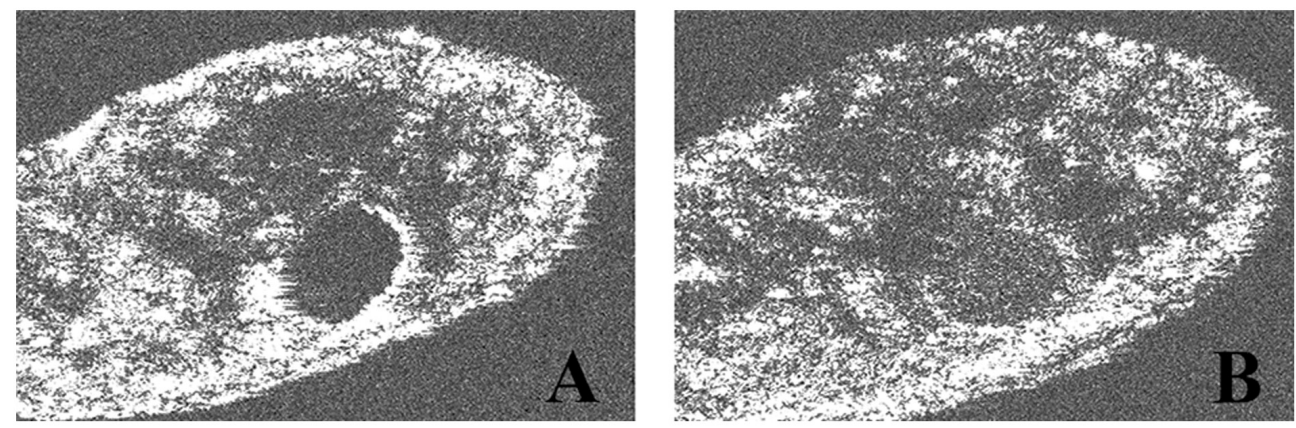

Figure 7. OCT analysis of the same section from Sample 6 before and after the non-conventional irrigation protocols. (A) Initial OCT, (B) +NanoCare Plus.

suggesting good diffusion capacity of these endodontic irrigants. This observation can lead to improvement of the classical irrigation protocols used in daily endodontic practice, as the apical area is the most refractory area to cleaning during treatment, and the presence of the nanoparticles in close contact with the root canal outline, as observed in the present study, may lead to improved disinfection efficiency of endodontic irrigants.

The results obtained in the present study are similar with literature data and show that the addition of gold and silver nanoparticles in root canal irrigants increases their optical properties. It is known that gold addition decreases the micro-infiltration at the root canal walls level, increasing the adhesion of filling materials to dentin. Both types of nanoparticles have proved antimicrobial efficiency. Furthermore, this property enhances the capacity of the irrigation protocol to reduce the number of remnant bacteria in the root canal lumen (Figs. 5-7).

One of the limitations of the present study could be the resolution of the OCT investigating system; because of the lack of a clear differentiation between the tubular dentin and the gold and silver nanoparticles the present study cannot demonstrate the penetration capacity of the nanoparticles at a tubular level. A better resolution of the OCT investigating system to differentiate between the dentin optical opacity and the one of the nanoparticles or the performance of other imagistic analysis such as Electronic Transmission Microscopy could offer more precise results.

Micro-infiltration tests performed on cleaned, shaped, and filled root canals after irrigation with solutions supplemented with nanoparticles can be performed in future studies, to prove the influence of these particles on the adhesion of various materials to root canal dentin, as shown in the literature (18-20). In addition, the present study can be continued and improved by investigating the efficacy of these irrigants on specific bacteria including E. faecalis or other species colonizing the root canal, to prove their antibacterial effect $(9,10,21)$.

The present study proved that gold and silver nanoparticles introduced in root canal irrigants could be evidenced through OCT imagistic analysis because of their optical opacity, different from that of the root canal dentin and from the empty root canal lumen. The optical opacity of these nanoparticles allows their ability to be highlighted in an empty root canal lumen, after the endodontic treatment is performed and the root canal is cleaned and shaped using specific protocols. There 
was no possibility to separately highlight gold from silver nanoparticles using this technique because both irrigants used in the present study contain both types of nanoparticles, with a bigger gold concentration for the NanoCare Gold irrigant.

The OCT investigation performed in the present study proved that the highest optical opacity in the investigated samples was obtained after using an alternation of these two irrigants, explained by a higher concentration of nanoparticles remaining in the root canal lumen.

Numerous applications of nanomedicine and the promising results of research regarding the applicability of nanotechnology in clinical medicine are prerequisites for the continuous development of this area (22-25).

Therefore, nanotechnology is currently one of the future directions for investigation in endodontics as well.

\section{Acknowledgements}

Not applicable.

\section{Funding}

No funding was received.

\section{Availability of data and materials}

The datasets used/analyzed in this study are available from the corresponding author, upon reasonable request.

\section{Authors' contributions}

FT, LMN, DR, MB and RC participated in data acquisitions and interpretation thereof. AP, MLN, VFD, CS, AM and SIS participated in the study design. LEC, FT, MB and LMN performed the statistical analysis. LMN, MB and FT are responsible for confirming the authenticity of the raw data. All authors drafted the manuscript. All authors read and approved the final version of the manuscript.

\section{Ethics approval and consent to participate}

Not applicable.

\section{Patient consent for publication}

Not applicable.

\section{Competing interests}

The authors declare that they have no competing interests.

\section{References}

1. Campos PI, La Fuenteha J, Tenorio RF and Acosta TL: Biocompatible antimicrobial irrigants and nanoparticles-sealers for endodontics. Entreciencias 1: 9-28, 2013.

2. Kim JS, Kuk E, Yu KN, Kim JH, Park SJ, Lee HJ, Kim SH, Park YK, Park YH, Hwang CY, et al: Antimicrobial effects of silver nanoparticles. Nanomedicine 10: e1119, 2014.

3. Hiraishi N, Yiu CK, King NM, Tagami J and Tay FR: Antimicrobial efficacy of $3.8 \%$ silver diamine fluoride and its effect on root dentin. J Endod 36: 1026-1029, 2010.
4. Peng G, Tisch U, Adams O, Hakim M, Shehada N, Broza YY, Billan S, Abdah-Bortnyak R, Kuten A and Haick H: Diagnosing lung cancer in exhaled breath using gold nanoparticles. Nat Nanotechnol 4: 669-673, 2009.

5. Baptista P, Pereira E, Eaton P, Doria G, Miranda A, Gomes I, Quaresma P and Franco R: Gold nanoparticles for the development of clinical diagnosis methods. Anal Bioanal Chem 391: 943-950, 2008

6. Kumar A, Bhargavi Mazinder B, Liang XL and Cui D: Gold nanoparticles: Promising nanomaterials fo the diagnostic of cancer and HIV/AIDS. J Nanomater 2011: 1-17, 2011.

7. Rayavarapu RG, Petersen W, Ungureanu C, Post JN, van Leeuwen TG and Manohar S: Synthesis and bioconjugation of gold nanoparticles as potential molecular probes for light-based imaging techniques. Int J Biomed Imaging 2007: 29817, 2007.

8. Rosi NL and Mirkin CA: Nanostructures in biodiagnostics. Chem Rev 105: 1547-1562, 2005.

9. Sofi W, Gowri M, Shruthilaya M, Rayala S and Venkatraman G: Silver nanoparticles as an antibacterial agent for endodontic infections. BMC Infect Dis 12: 60, 2012.

10. Grade S, Eberhard J, Jakobi J, Winkel A, Stiesch S and Barcikowski S: Alloying colloidal silver nanoparticles with gold disproportionally controls antibacterial and toxic effects. Gold Bull 47: 83-93, 2014.

11. Mackiewicz A and Olczak-Kowalczyk D: Microscopic evaluation of surface topography and chemical composition of Nanocare Gold. J Stomatol 67: 826-840, 2014.

12. Idoraşi L, Cîrligeriu L, Sinescu C, Zaharia C, Stan AT and Negruţiu ML: Silver nanotechnology-the future in caries therapy? A report of two cases. J Interdiscip Med 2: 67-71, 2017.

13. Huang D, Swanson EA, Lin CP, Schuman JS, Stinson WG, Chang W, Hee MR, Flotte T, Gregory K, Puliafito CA, et al: Optical coherence tomography. Science 254: 1178-1181, 1991.

14. Sinescu C, Marsavina L, Negrutiu ML, Rusu LC, Ardelean L, Rominu M, Antoniac I, Topala FI and Podoleanu A: New metallic nanoparticles modified adhesive used for time domain optical coherence tomography evaluation of class II direct composite restoration. Rev Chim 63: 380-383, 2012.

15. Craciunescu MC, Negrutiu ML, Hogea E, Freiman PC, Boariu M, Craciunescu E and Sinescu C: Bacteriostatic effect of silver nanoparticles over acrylic resin and composite dental materials. Mater Plast 51: 414-416, 2014.

16. Schneider CA, Rasband WS and Eliceiri KW: NIH image to ImageJ: 25 years of image analysis. Nat Methods 9: 671-675, 2012.

17. Abramoff MD, Magalhães PJ and Ram SJ: Image processing with ImageJ. Biophoton Int 11: 36-42, 2004.

18. Kesler Shvero D, Abramovitz I, Zaltsman N, Perez Davidi M, Weiss EI and Beyth N: Towards antibacterial endodontic sealers using quaternary ammonium nanoparticles. Int Endod J 46: 747-754, 2013.

19. Pagonis TC, Chen J, Fontana CR, Devalapally H, Ruggiero K, Song X, Foschi F, Dunham J, Skobe Z, Yamazaki H, et al: Nanoparticle-based endodontic antimicrobial photodynamic therapy. J Endod 36: 322-328, 2010.

20. Moghadas L, Shahmoradi M and Narimani T: Antimicrobial activity of a new nanobased endodontic irrigation solution: In vitro study. Dent Hypotheses 3: 142-146, 2012.

21. Van Dong P,Ha CH, Binh LT and Kasbohm J: Chemical synthesis and antibacterial activity of novel-shaped silver nanoparticles. Int Nano Lett 2: 9, 2012.

22. Kovvuru SK, Mahita VN, Manjunatha BS and Babu BS: Nanotechnology: The emerging science in dentistry. J Orofac Res 2: 33-36, 2012.

23. Verma SK, Prabhat KC, Goyal L, Rani M and Jain A: A critical review of the implication of nanotechnology in modern dental practice. Natl J Maxillofac Surg 1: 41-44, 2010.

24. Martinez-Gutierrez F, Olive PL, Banuelos A, Orrantia E, Nino N, Sanchez EM, Ruiz F, Bach H and Av-Gay Y: Synthesis, characterization, and evaluation of antimicrobial and cytotoxic effect of silver and titanium nanoparticles. Nanomedicine 6: 681-688, 2010

25. Cheon JY, Kim SJ, Rhee YH, Kwon OH and Park WH: Shape-dependent antimicrobial activities of silver nanoparticles. Int J Nanomedicine 14: 2773-2780, 2019.

This work is licensed under a Creative Commons Attribution-NonCommercial-NoDerivatives 4.0 International (CC BY-NC-ND 4.0) License. 